\title{
Measuring the dynamical critical exponent of an ordering alloy using x-ray photon correlation spectroscopy
}

\author{
Frédéric Livet ${ }^{1 *}$, Mathieu Fèvre ${ }^{2}$, Guillaume Beutier ${ }^{1}$, \\ Federico Zontone ${ }^{3}$, Yuriy Chushkin ${ }^{3}$, and Mark Sutton ${ }^{4}$ \\ 1 Univ. Grenoble Alpes, CNRS, SIMAP, F-38000 Grenoble, France \\ 2 Laboratoire d'Etude des Microstructures, \\ UMR 104 CNRS-ONERA, BP 72, 92322 Châtillon, France \\ 3 ESRF - The European Synchrotron, \\ 71 Avenue des Martyrs, Grenoble, France and \\ ${ }^{4}$ Center of the Physics of Materials, McGill University, \\ 3600 University street, Montreal, PQ, Canada, H3A-2T8*
}

(Dated: May 25, 2018)

\begin{abstract}
The dynamics of the order fluctuations in the $\mathrm{AuAgZn} \mathrm{n}_{2}$ close to the critical point $\left(T_{c} \simeq 609\right.$ K) was observed by coherent x-ray scattering. With the high beam intensity of the ID10 ESRF beamline and with the new pixel detector, the dynamics was measured with a few tens of millisecond resolution. The intensity connected to the diffuse scattering corresponding to fluctuations was unambiguously distinguished from the surface pretransitional ordering occurring in this system close to $\mathrm{T}_{c}$. The variations of the fluctuation time with temperature and wave vectors were measured in this system belonging to the universality class of Ising second order transition with a non-conserved order parameter. The direct observation of the critical slowing down in the vicinity of the second-order transition led to an estimate of the dynamic exponent $z \simeq 1.96(11)$, in rough agreement with theory (model "A" of Ref. 1).
\end{abstract}




\section{INTRODUCTION}

X-ray photon correlation spectroscopy (XPCS) has raised considerable interest to probe equilibrium and non-equilibrium fluctuations in soft and hard systems [2-4] at the nanoscale and on timescales increasingly smaller $[5,6]$. In alloys, the technique was used e.g., to study domain formation [7-9] or phason fluctuations [10]. In $\mathrm{AuAgZn}_{2}$, a second order transition occurs at $609 \mathrm{~K}[11,12]$. Above this critical temperature $T_{c}$, the alloy has a simple cubic $\mathrm{B} 2$ structure where $\mathrm{Au}$ and $\mathrm{Ag}$ atoms share the same simple cubic atomic sites. Below $T_{c}$, the alloy has a face centered cubic $\mathrm{L} 21_{1}$ structure, as shown in Fig. 1. This transition ("Heusler") is equivalent to a SC antiferromagnetic Ising system [13]. In the vicinity of the critical temperature, the fluctuation length $\xi$ is observed to increase to $\xi \gtrsim 500 \AA$, with the scaling law: $\xi \simeq 1.76 \times\left(\left(T-T_{c}\right) / T_{c}\right)^{-\nu}$, with $\xi$ in Anströms $(\AA)$ and $\nu=0.63$, the classical Ising value.

As ordering requires only atomic jumps between neighboring sites, the $\mathrm{AuAgZn}$ alloy can be considered as a model system to investigate second order transitions with "non conserved order" parameters i.e., the "A" model of Hohenberg and Halperin [1, 14]. Experiments are carried out in a small q-range in the vicinity of the $\frac{1}{2} \frac{1}{2} \frac{1}{2}$ superstructure peak.

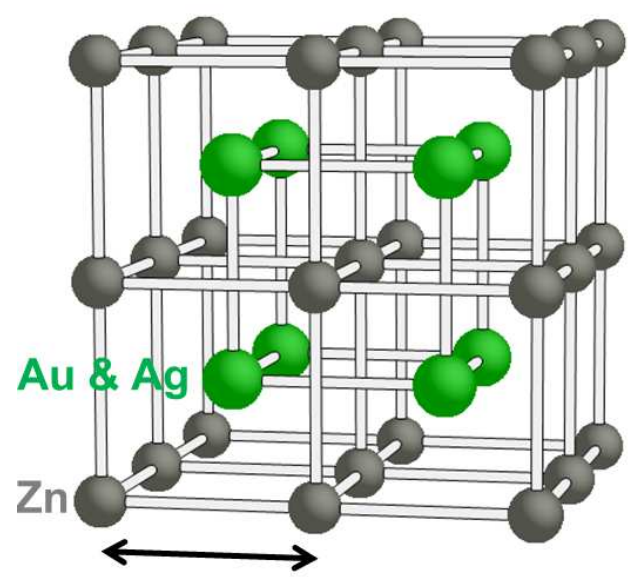

a

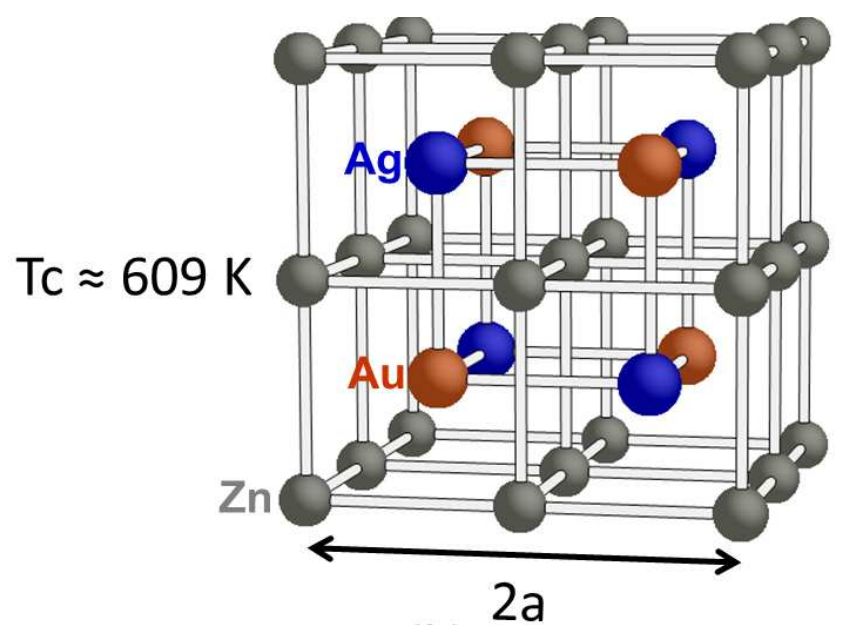

(b)

FIG. 1. (a) B2 and (b) $\mathrm{L} 2{ }_{1}$ ordered structures of the $\mathrm{AuAgZn} \mathrm{n}_{2}$ alloy near $\mathrm{T}=609 \mathrm{~K}$. The lattice parameter $a$ of the B2 phase is close to $3.17 \AA$ at $603 \mathrm{~K}$.

Outside the critical region, the driving force of the dynamics is the interface motion between ordered domains. The domain size $L$ increases according to the diffusion law $L=\sqrt{D t}$, 
which applies for non-conserved dynamics (see e.g., Ref. 15). In a previous experiment [16], the diffusion coefficient $D$ in $\mathrm{AuAgZn}{ }_{2}$ was estimated to be $5 \times 10^{5} \AA^{2} s^{-1}$ at $\mathrm{T}=607 \mathrm{~K}$. This led to an elementary diffusion time $\tau_{0} \simeq 20 \mu$ s corresponding to atomic jumps between two sites of the Au-Ag simple cubic sublattice (see Fig. 1b). In a quenched sample with antiphase domains, speckles are observed $[16,17]$ and changes in the speckle pattern close to $T_{c}$ reflect the microscopic time evolution of the fluctuations.

In the critical region, equilibrium fluctuations have a characteristic relaxation time $\tau$ and, from the dynamic renormalization model[1], $\tau$ is related to $\xi$ and $q$ by the following expression:

$$
\tau(q, T) \propto \xi^{z} \Phi(q \xi) \propto \begin{cases}\left|\left(T-T_{c}\right) / T_{c}\right|^{-z \nu} & \text { for } q \xi \ll 1 \\ q^{-z} & \text { for } q \xi \gg 1\end{cases}
$$

where the wave vector $q$ corresponds to the distance in the reciprocal lattice to the superstructure Bragg position. Dynamic renormalization group calculations [14], [18] yield $z \simeq 2.02$. The experimental characterization of the critical fluctuation dynamics is a real challenge since in this system, as explained in Ref. 16, heating devices providing high temperature stability (a few $\mathrm{mK}$ ) and very good accuracy $\left(\Delta q \lesssim 10^{-4} \AA^{-1}\right)$ are requested.

Pioneering experiments on the dynamics of critical fluctuations were attempted by Brauer et al. [19] in the $\mathrm{Fe}_{3} \mathrm{Al}$ binary alloy which exhibits a $\mathrm{B} 2-\mathrm{DO}_{3}$ second order transition when the temperature is close to $824 \mathrm{~K}$. In our previous measurements [16], only correlation functions at a fixed temperature and in a narrow q-range could be measured. The measurement of reliable time correlation functions whose behavior can be compared to Renormalization Group calculations or numerical simulations is still missing.

In this article, some significant improvements in the characterization of critical fluctuation dynamics in an ordering alloy are outlined and the first measurement of the related dynamical critical exponents $z$ is reported.

\section{EXPERIMENTAL DETAILS}

Measurements were carried out at the ID10 beamline of the ESRF (France). Undulators were tuned to produce $8 \mathrm{keV}$ photons and a $\mathrm{Si}(111)$ monochromator was used to select a wavelenth of $1.55 \AA$ with an intrinsic energy resolution $\Delta E / E \simeq 1.4 \times 10^{-4}$. The incident beam was focused with Beryllium lenses to have a $3.5 \mu \mathrm{m}(\mathrm{v}) \times 20 \mu \mathrm{m}(\mathrm{h})$ section at 
the sample position and a partially coherent flux of $\simeq 2 \times 10^{10}$ photons per second. The (111) oriented sample surface was tilted by about $12^{\circ}$ to observe the scattering intensities relative to the $\frac{1}{2} \frac{1}{2} \frac{1}{2}$ superstructure reflection of the B2 structure, with a vertical diffraction plane. The gauge volume probed by the x-ray beam was thus close to $17 \mu \mathrm{m} \times 20 \mu \mathrm{m} \times 1 \mu \mathrm{m}$; the latter value being the x-ray penetration depth into the tilted $\mathrm{AuAg} \mathrm{Zn}_{2}$ sample. A 2D pixel detector [20] with $516 \times 516$ pixels of $55 \mu \mathrm{m} \times 55 \mu \mathrm{m}(2 \times 2$ Medipix2 chips $)$ was placed at a distance of $1.73 \mathrm{~m}$ from the sample position. The q-resolution of one pixel was thus $1.29 \times 10^{-4} \AA^{-1}$. Measurements were performed in the vertical diffraction plane with a detector angle $2 \theta_{B}$ close to $24^{\circ}$. The results described in the following were obtained by recording sets of 40,000 frames with a maximum time spacing between frames of $0.02 \mathrm{~s}$. The temperature control of the sample was performed with a Lakeshore controller and a small furnace in vacuum developed to have a maximum deviation of a few $\mathrm{mK}$ from a fixed temperature between $553 \mathrm{~K}$ and $638 \mathrm{~K}$. The $\mathrm{AuAgZn}_{2}$ samples were cylinders with a diameter of $8 \mathrm{~mm}$ and a height of $\simeq 1-2 \mathrm{~mm}$. Two $70 \AA$ thick aluminum layers were deposited on the sample surfaces to prevent losses of zinc during heating (see detail in Ref. 16). The coherence contrast $\beta$ of this setup was estimated from the observation of speckles in a quenched sample [16, 21] to $3.5 \%$.

Well above $T_{c}$ an isotropic diffuse intensity was recorded at the ordering reflection position. Close to $T_{c}$, some pretransitional scattering (PTS) was observed, as shown in Fig. 2(a). This scattering is cross-shaped: in the $q_{x}$ direction (horizontal in the figure), some scattering corresponding to surface mosaicity is observed and in the $q_{y}$ direction, the scattering is elongated perpendicular to the surface, corresponding to the small thickness of the surface ordering.

PTS was strongly temperature-dependent even when only a few $\mathrm{mK}$ variations of the temperature did occur. For large scattering vectors, the intensity appeared isotropic, but the fluctuation dynamics was too fast and the intensity too low to obtain reliable results. For all these reasons, only a part of the recorded intensities was utilized for data processing. This was done by using masks like the one represented in Fig. 2(b), where data in black were discarded. After an angular average, the intensity profile of each diffraction pattern was separated into two contributions using [16]: 


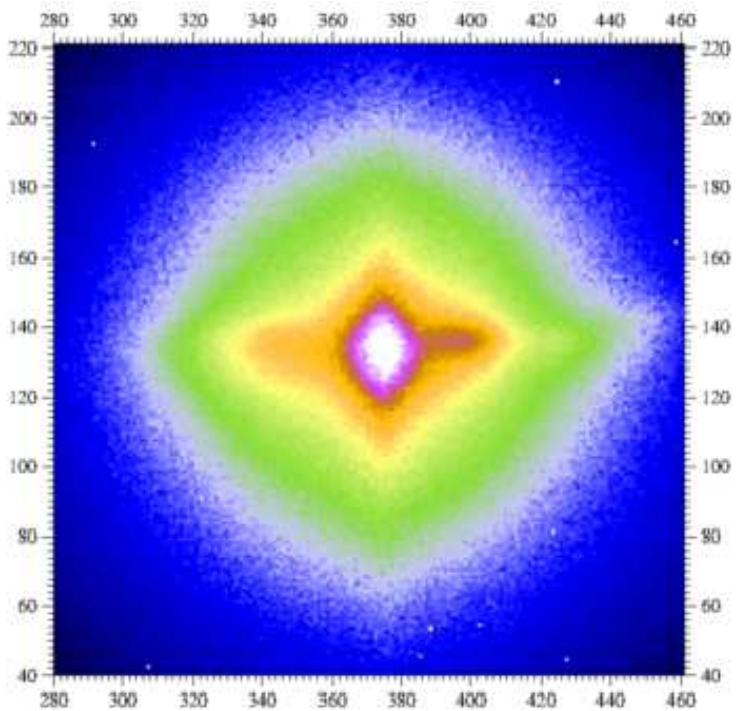

(a)

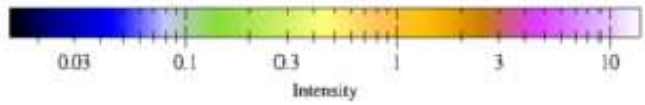

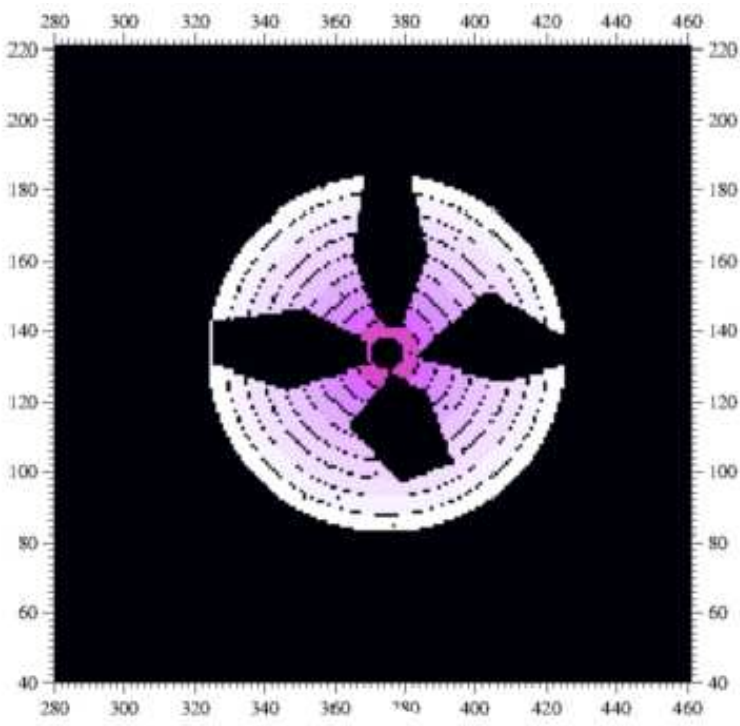

(b)

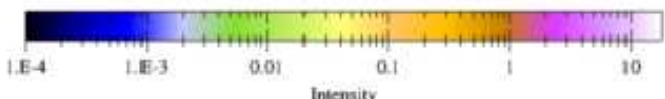

FIG. 2. (a) Measured scattering at $609.35 \mathrm{~K}\left(\mathrm{~T}_{c}+0.188 \mathrm{~K}\right)$ and (b) the mask used and the typical circular rings where the dynamics was oberved. Both figures show the same $100 \times 100$ pixel area, each pixel is $1.29 \times 10^{-4} \AA^{-1}$

$$
I(q)=\frac{S(q=0)}{1+(q \xi)^{1.97}}+\frac{B}{\left(1+(q L)^{2}\right)^{2}}
$$

The first term in Eq. 2 is a very good approximation of the static critical scattering $S(q)$ and the second term roughly fits to PTS. PTS is strongly peaking and exhibits some anisotropy even after masking for the smallest q-values (qi0.0008 $\AA^{-1}$ ) where the fit is poor. This second term is introduced because for intermediate q-values the scattering has a $q^{-4}$ behavior, which means that PTS corresponds to small well defined ordered domains.

As shown in Fig. 3, the analysis of the temperature behavior of $S(q=0)$ and $\xi$ in the vicinity of $T_{c}$ led to a reliable estimate of the critical temperature. $T_{c}$ was estimated to $609.162(15) \mathrm{K}$ ( or $336.012^{\circ} \mathrm{C}$ ), and its variations was less than $0.02 \mathrm{~K}$ during the 48 hours of the measurements. Our results were in a good agreement with the theoretical values $\gamma=1.241$ and $\nu=0.63[22]$.

The dynamics was characterized from the intensity autocorrelation function $g_{2}(q, t)$ calculated by a time average and an angular average [16] over circular domains of thickness $\Delta q$ 

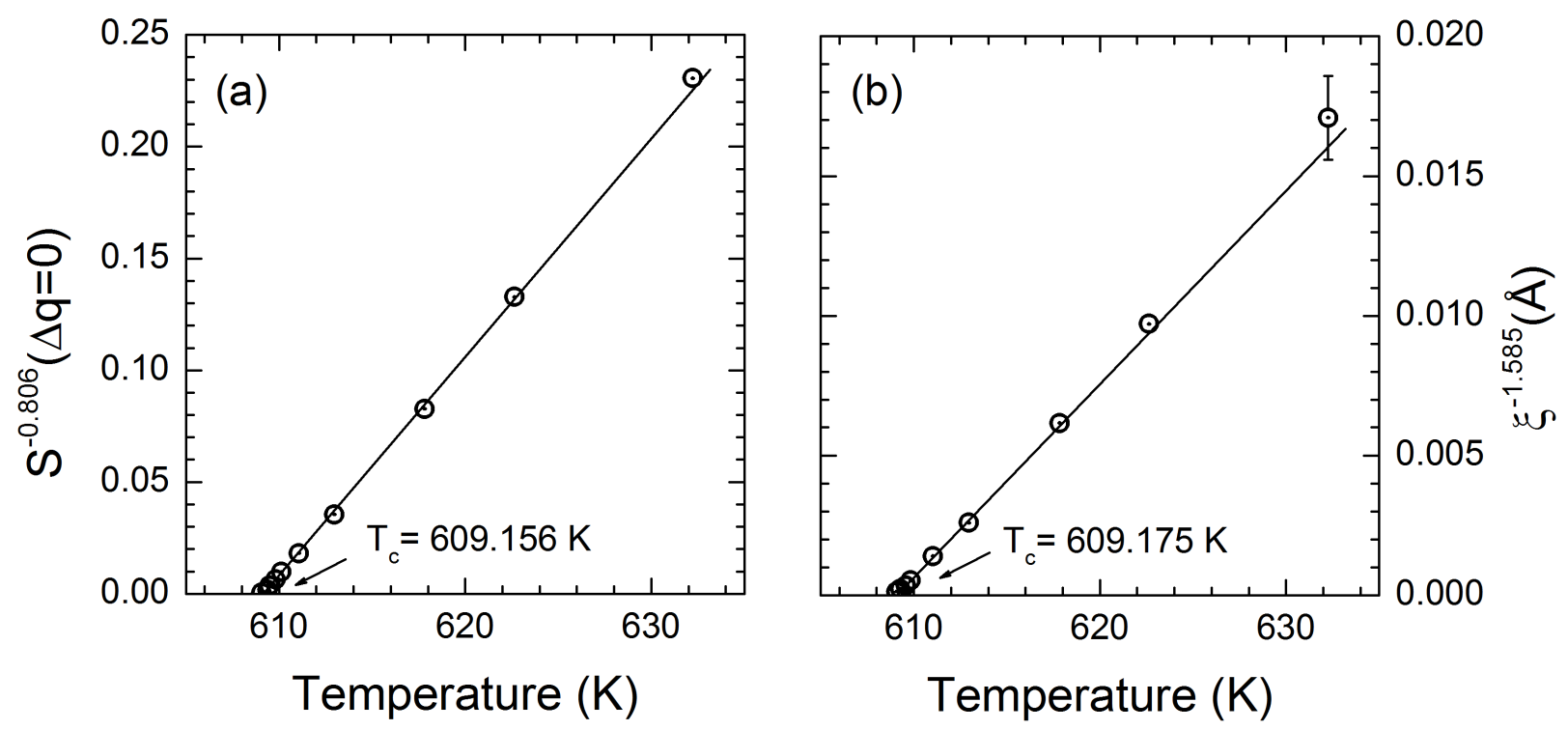

FIG. 3. Determination of the critical temperature by fitting intensity profiles to Eq. 2. (a) from the variation of $S^{-1 / \gamma}(q=0)$ with $\gamma=1.241$. (b) from the variation of $\xi^{-1 / \nu}$ with $\nu=0.63$.

around the Bragg position as shown in Fig. 2(b)). A dedicated program for XPCS written with python by one of us (Y.C.) was used for calculating $g_{2}$.

\section{RESULTS}

The correlation functions $g_{2}(q, t)$

Fig. 4 represents the values of the time correlation functions $\mathrm{g}_{2}(q, t)$ obtained for a set of $q$-vectors at a fixed temperature $T_{c}+0.113$. The $\mathrm{g}_{2}(q, t)$ functions calculated for a given temperature, were fitted by the following expression:

$$
g_{2}(q, t)=1+a_{0}+b_{0} e^{-2 t / \tau(q)}
$$

where $\tau(q)$ is the characteristic relaxation time of the critical fluctuations, $a_{0}$ is essentially due to the anisotropy of the PTS (see Fig. 2) and, $b_{0} \simeq \beta x^{2}$ is connected to the coherence $\beta \simeq 0.035$ and to $x$, the ratio of the critical to PTS intensities. Equation 3 assumes that heterodyne interferences between the two scattering in Eq. 2 were negligible. $b_{0}$ increases with increasing values of $q$ : in Fig. 4 , its value is in the 0.02-0.004 range. The results of the least-square fitting using Eq. 3 correspond to the solid lines in Figs. 4. Owing to the high 


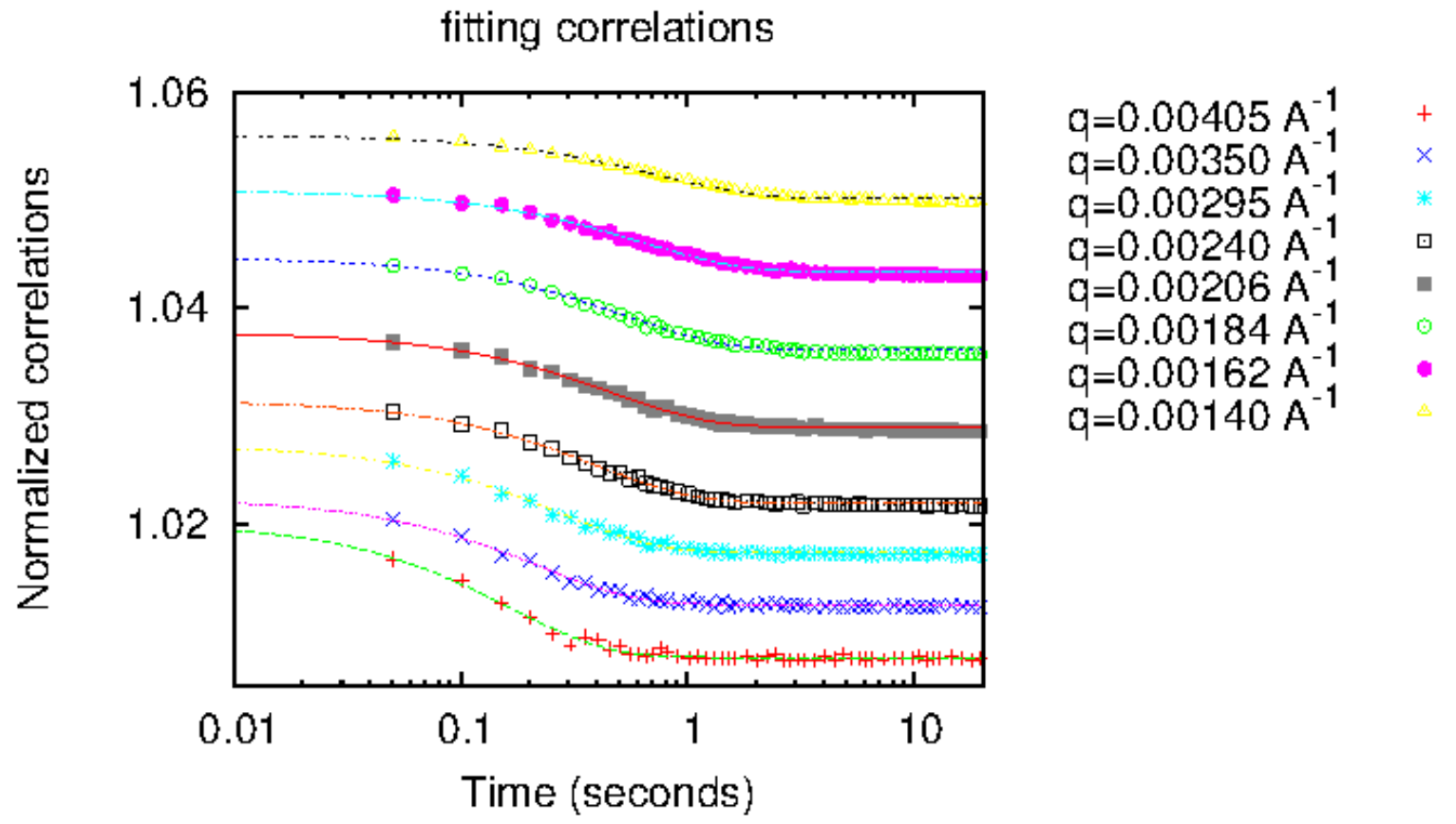

FIG. 4. Time correlations obtained for various $q$ vectors at $T=T_{c}+0.113 \mathrm{~K}$. Curves are shifted vertically for sake of visibility. Symbols correspond to values of the $g_{2}(q, t)$ function and solid lines to a least-square fits with Eq. 3

experimental stability of the beamline, a reasonable accuracy was obtained for $q \geq 10^{-3} \AA^{-1}$. For lower $q$ values, $b_{0}$ was too small $\left(b_{0}<10^{-3}\right)$ and some experimental issues prevented from obtaining reliable results. For measurements performed at $\mathrm{T}-\mathrm{T}_{c} \mathrm{i} 0.2 \mathrm{~K}$, oscillations of the $\mathrm{g}_{2}(q, t)$ correlation functions for $q<0.0008 \AA^{-1}$ were caused by the high sensiblity of the PTS to small temperature instabilities (fluctuations of $\pm 2 \mathrm{mK})$. Long term $(t>100 \mathrm{~s}$ ) instabilities of the experiment were also observed.

\section{Critical fluctuation relaxation times $\tau(q, T)$ and $\tau_{0}(T)$}

The characteristric relaxation times $\tau(q, T)$ in Eq. 3 deduced from the intensity autocorrelation calculated at different temperatures $T$ and $q$-vectors are plotted in Fig. 5(a). The shortest values of $\tau$ close to $0.1 \mathrm{~s}$ were measured with a good accuracy well above $T_{c}$ (i. e. $\left.T_{c}+0.7 \mathrm{~K}\right)$ and for large $q$ values (i. e. $q \simeq 0.006 \AA^{-1}$ ). The largest $\tau$ values are close to $10 \mathrm{~s}$ and were obtained from measurements at small $q$ values (i.e. $q \simeq 0.0006 \AA^{-1}$ )) in the vicinity 

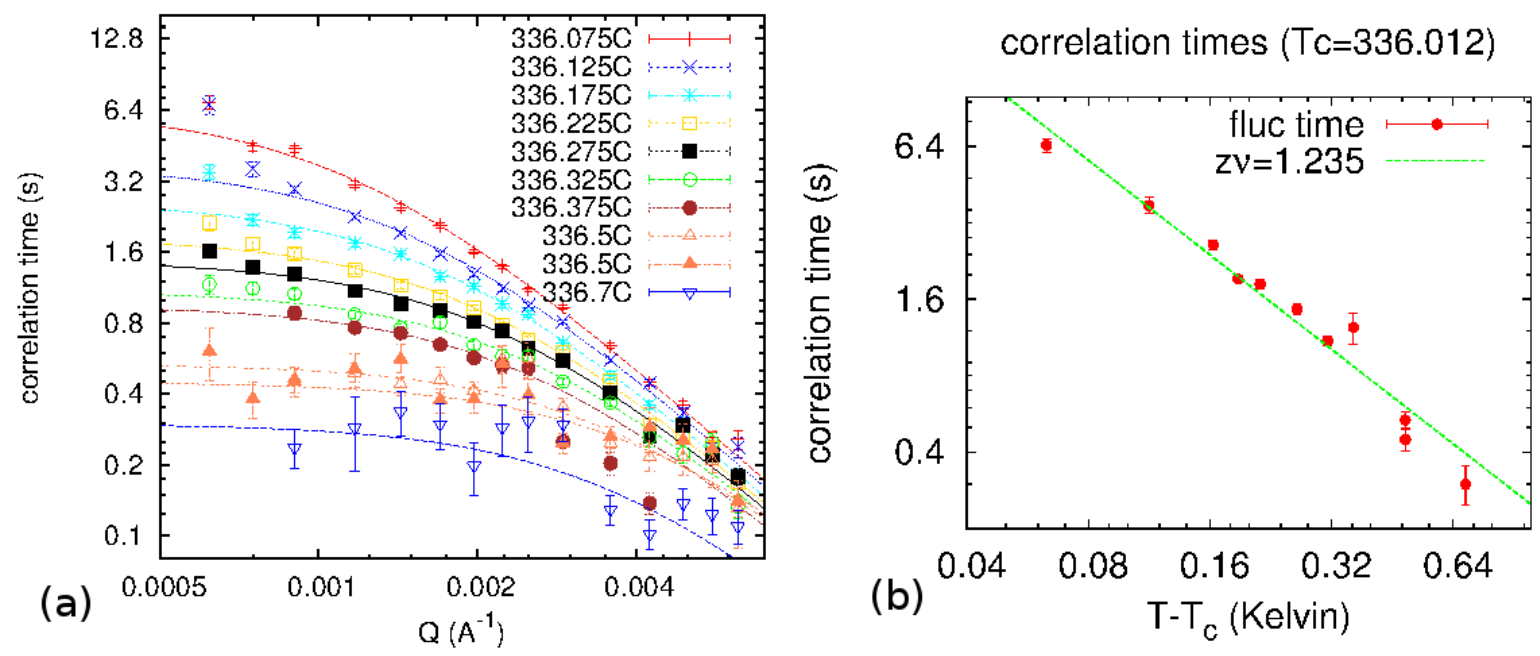

FIG. 5. (a) Fluctuation times $\tau(q, T)$ for various temperatures and the lorentzian fits. (b) Temperature variations of $\tau_{0}(T)$. An estimate of the exponent yielded $z \nu=1.235(70)$

of $\mathrm{T}_{c}$, in a domain where large PTS was measured. The temperature dependent fluctuation time $\tau_{0}(T)$ (for $q=0$ ) was determined using least-squares fits with the following equation:

$$
\tau(q, T)=\tau_{0}(T) /\left(1+\left(q / q_{0}\right)^{2}\right)
$$

Equation 4 assumes a Lorentzian $q$-dependence of $\tau(q)$ (the solid lines in Fig. 5(a)), compatible with mean-field approximations (i. e. $z=\gamma / \nu=2$ ). The double logarithmic plot of $\tau_{0}(T)$ shown in Fig. 5(b), roughly corresponds to a scaling law:

$$
\tau_{0}(T)=\left(\left(T-T_{c}\right) / T_{c}\right)^{\nu z}
$$

with $\nu z=1.235(70)$. From $\nu=0.630$ [22], we estimate $z \simeq 1.96(11)$. This value is in agreement with the best theoretical value of 2.017 [18], but with a poor accuracy.

\section{DISCUSSION}

The $\mathrm{AuAgZn} \mathrm{n}_{2}$ alloy is an example for the study of the "model A" dynamics of phase transitions, where the order parameter is changed without long range diffusion, analogous to a local spin flip in the Ising model. In order to carry out an XPCS study at large angle, a short x-ray penetration depth is mandatory and the strong contrast between $\mathrm{Au}$ and $\mathrm{Ag}$ atoms provides a reasonable intensity close to the ordering Bragg peak. This penetration 
depth $\left(\mu^{-1} \simeq 10 \mu \mathrm{m}\right)$ leads to small path length differences of the beam in the sample $(L \simeq$ $2 . \mu^{-1} \sin ^{2}\left(\theta_{B}\right) \simeq 0.9 \mu \mathrm{m}$ is nevertheless significantly larger than the longitudinal coherence length of the experiment $\left(\Lambda_{L}=\lambda^{2} /(2 . \delta \lambda) \simeq 0.5 \mu \mathrm{m}\right)$.

Main difficulty is in dealing with PTS, which makes results in the close vicinity of $T_{c}$ (in Fig.4, temperatures $T<T_{c}+0.07 \mathrm{~K}$ were discarded) and for very small angles $(q<$ 1. $\times 10^{-3} \AA^{-1}$ ) unreliable. PTS is connected to the two $70 \AA$ thick surface layers which were deposited in order to prevent zinc evaporation. While these layers are an excellent chemical barrier, they probably add some constraints that favor surface ordering. As a result, better results in the dynamical study were obtained for regions where the scattering is small. As PTS is connected to surface defects, its interferences with the critical scattering from the inside of the sample is here assumed negligible. For this reason, heterodyning has been neglected, contrary to our previous paper (see 16).

A measurement of the dynamical critical exponent $z \simeq 1.96(11)$ of a 3D Ising system was performed using the XPCS method. The precision of the result does not allow a distinction from mean-field theory (which yields $z=2$ ), and this makes valid the approximations used here: an exponential in Eq.3 and a Lorentzian for $\Phi(x)$ in Eq.4.

Obviously, these experiments will be significantly eased with the next ESRF upgrade, where the source brillance should be increased more than one order of magnitude. This will bring a large improvement in the measurement of the dynamic exponent $z$.

The authors want to thank the experimental team of the ID10 beamline of ESRF.

* frederic.livet@simap.grenoble-inp.fr

[1] P. C. Hohenberg and B. I. Halperin, Rev. Mod. Phys. 49, 436 (1977).

[2] S. B. Dierker, R. Pindak, R. M. Fleming, I. K. Robinson, and L. Berman, Phys. Rev. Lett. 75, 449 (1995).

[3] F. Livet, F. Bley, R. Caudron, E. Geissler, D. Abernathy, C. Detlefs, G. Grübel, and M. Sutton, Phys. Rev. E 63, 036108 (2001).

[4] H. Kim, A. Rühm, L. Lurio, J. Basu, J. Lal, S. Mochrie, and S. Sinha, Materials Science and Engineering: C 24, 11 (2004).

[5] G. Grübel, C. R. Physique 9, 668 (2008). 
[6] O. G. Shpyrko, Journal of Synchrotron Radiation 21, 1057 (2014).

[7] K. Ludwig, F. Livet, F. Bley, J. P. Simon, R. Caudron, D. Le-Bolloc'-h, and A. Moussaid, Phys. Rev. 72, 144201 (2005).

[8] L-M. Stadler, B. Sepiol, J. W. Kantelhardt, I. Zizak, G. Grübel, and G. Vogl, Phys. Rev. B 69, 224301 (2004).

[9] A. Fluerasu, M. Sutton, and E. M. Dufresne, Phys. Rev. Lett. 94, 055501 (2005).

[10] S. Francoual, F. Livet, M. de Boissieu, F. Yakhou, F. Bley, A. Létoublon, R. Caudron, and J. Gastaldi, Phys. Rev. Lett. 91, 225501 (2003).

[11] M. E. Brooke and R. W. Smith, Scr. Metall. 3, 667 (1969).

[12] L. Muldawer, J. Appl. Phys. 37, 2062 (1966).

[13] F. Livet, F. Bley, J. P. Simon, R. Caudron, J. Mainville, M. Sutton, and D. Lebolloc'h, Phys. Rev. 66, 134108 (2002).

[14] R. Folk and G. Moser, J. Phys. A: Math. Gen. 39, R207 (2006).

[15] A. J. Bray, Advances in physics 43, 357 (1994).

[16] F. Livet, M. Fèvre, G. Beutier, and M. Sutton, Phys. Rev. B 92, 094102 (2015).

[17] M. Sutton, S. G. J. Mochrie, T. Greytak, S. E. Nagler, L. E. Berman, G. E. Held, and G. B. Stephenson, Nature 352, 608 (1991).

[18] V. V. Prudnikov, A. V. Ivanov, and A. A. Fedorenko, JETP Lett. 66, 835 (1997).

[19] S. Brauer, G. B. Stephenson, M. Sutton, R. Bruning, E. Dufresne, S. G. J. Mochrie, G. Grübel, J. Als-Nielsen, and D. L. Abernathy, Phys. Rev. Lett. 74, 2010 (1995).

[20] R. de Vries, S. Weijers, K. Bethke, V. Kogan, J. Vasterink, A. Kharchenko, M. Fransen, and J. Bethke, Nucl. Instrum. Methods Phys. Res. A 576, 164 (06 2007).

[21] F. Livet and M. Sutton, Comptes Rendus Physique 13, 227 (2012).

[22] J. C. LeGuillou and J. Zinn-Justin, Physical Review B 21, 3976 (1980). 